\title{
Characterization of FliL Proteins in Bradyrhizobium diazoefficiens: Lateral FliL Supports Swimming Motility, and Subpolar FliL Modulates the Lateral Flagellar System
}

\author{
Florencia Mengucci, a Carolina Dardis, a Elías J. Mongiardini,a María J. Althabegoiti, ${ }^{a}$ Jonathan D. Partridge,b (D) Seiji Kojima, \\ Michio Homma, ${ }^{c}$ Juan I. Quelas, ${ }^{\text {** }}$ Aníbal R. Lodeiro ${ }^{a, d}$ \\ aLaboratorio de Interacciones entre Rizobios y Soja (LIRyS), IBBM, Facultad de Ciencias Exactas, Universidad Nacional de La Plata y CCT-La Plata CONICET, La Plata, \\ Argentina \\ bDepartment of Molecular Biosciences, University of Texas at Austin, Austin, Texas, USA \\ ‘Division of Biological Science, Graduate School of Science, Nagoya University, Nagoya, Japan \\ ¿Laboratorio de Genética, Facultad de Ciencias Agrarias y Forestales, Universidad Nacional de La Plata, La Plata, Argentina
}

ABSTRACT Bradyrhizobium diazoefficiens is a soil alphaproteobacterium that possesses two evolutionarily distinct flagellar systems, a constitutive subpolar flagellum and inducible lateral flagella that, depending on the carbon source, may be expressed simultaneously in liquid medium and used interactively for swimming. In each system, more than 30 genes encode the flagellar proteins, most of which are well characterized. Among the exceptions is FliL, which has been scarcely studied in alphaproteobacteria and whose function in other bacterial classes is somewhat controversial. Because each $B$. diazoefficiens flagellar system contains its own fil paralog, we obtained the respective deletions $\Delta f_{\text {fil }}$ (subpolar) and $\Delta f_{i l} L_{L}$ (lateral) to study their functions in swimming. We determined that $\mathrm{FliL}_{\mathrm{L}}$ was essential for lateral flagellum-driven motility. Fli $L_{s}$ was dispensable for swimming in either liquid or semisolid medium; however, it was found to play a crucial role in upregulation of the lateral flagellum regulon under conditions of increased viscosity/flagellar load. Therefore, although $\mathrm{FliL}_{\mathrm{s}}$ seems to be not essential for swimming, it may participate in a mechanosensor complex that controls lateral flagellum induction.

IMPORTANCE Bacterial motility propelled by flagella is an important trait in most environments, where microorganisms must explore the habitat toward beneficial resources and evade toxins. Most bacterial species have a unique flagellar system, but a few species possess two different flagellar systems in the same cell. An example is Bradyrhizobium diazoefficiens, the $\mathrm{N}_{2}$-fixing symbiont of soybean, which uses both systems for swimming. Among the less-characterized flagellar proteins is FliL, a protein typically associated with a flagellum-driven surface-based collective motion called swarming. By using deletion mutants in each flagellar system's fliL, we observed that one of them (lateral) was required for swimming, while the other (subpolar) took part in the control of lateral flagellum synthesis. Hence, this protein seems to participate in the coordination of activity and production of both flagellar systems.

KEYWORDS Bradyrhizobium, FliL, flagella, swimming

radyrhizobium diazoefficiens, the $\mathrm{N}_{2}$-fixing symbiont of soybean, is an alphaproteobacterium that possesses two flagellar systems which evolved independently (1-3). This dual flagellar system comprises a single subpolar flagellum similar to the polar flagellum of Caulobacter crescentus and several lateral flagella similar to the flagella of Ensifer meliloti and Brucella spp. (3-5). Unlike other dual-flagellar-system bacterial
Citation Mengucci F, Dardis C, Mongiardini EJ, Althabegoiti MJ, Partridge JD, Kojima S, Homma M, Quelas JI, Lodeiro AR. 2020. Characterization of FliL proteins in Bradyrhizobium diazoefficiens: lateral FliL supports swimming motility, and subpolar FliL modulates the lateral flagellar system. J Bacteriol 202:e00708-19. https://doi.org/10 $.1128 / \mathrm{JB} .00708-19$.

Editor Anke Becker, Philipps-Universität Marburg

Copyright $\odot 2020$ American Society for Microbiology. All Rights Reserved.

Address correspondence to Juan I. Quelas, quelas@biol.unlp.edu.ar, or Aníbal R. Lodeiro, lodeiro@biol.unlp.edu.ar.

* Present address: Juan I. Quelas, Y-TEC, Berisso, Argentina.

Received 18 November 2019 Accepted 7 December 2019 Accepted manuscript posted online 16 December 2019

Published 11 February 2020 
species, in which the (sub)polar flagellum is used for swimming in liquid medium and the lateral flagella are used for swarming over wet surfaces $(6,7)$, in B. diazoefficiens, both flagellar systems are expressed simultaneously by planktonic cells in liquid medium $(2,4,8,9)$, a property that this bacterium shares only with Shewanella putrefaciens (10) among the bacterial species characterized to date. Thus, swimming behavior of $B$. diazoefficiens is an emergent property that results from interaction between both flagellar systems, which might enable these bacteria to better explore the complex soil pore space (2). Although under laboratory conditions $B$. diazoefficiens performs swarming motility, the lateral flagella are not strictly required for this phenotype, which seems unnecessary for displacement into the soil (11). Moreover, the high cell numbers required for swarming are seldom reached in the soil pore space, and thus swarming motility seems to be an unimportant trait for free-living $B$. diazoefficiens in its natural habitat formed by the soil pore space, the rhizosphere, and the root surfaces (2).

In B. diazoefficiens planktonic cells, the subpolar flagellum is expressed constitutively under the conditions explored so far, while the expression of lateral flagella, which is controlled by the class IA regulator regR and the class IB regulator lafR, requires certain carbon sources, oxic conditions, viscosity, or swimming in semisolid $0.3 \%$ agar medium $(4,5,11-13)$. The presence of lateral flagella in $B$. diazoefficiens cells that swim near surfaces seemingly prevents premature binding to the surface, which might allow swimming for longer distances than those possible for cells with only subpolar flagella (2). This property might be advantageous for dispersal into soil pores near the root surfaces in search for infectible root cells. Preliminary results also indicated that lateral flagella are better adapted for swimming in viscous medium (2).

Bacterial flagella are complex structures with the following three main components: the basal body inserted into the membrane system, which contains the flagellar motor and the export apparatus for secretion of the extracellular flagellar components; the flexible connector hook; and the helical filament that projects out of the cell and is several times longer than the cell body (see reference 5 for a scheme). Flagella are thus composed of many different structural proteins, with the functions of most of them known $(14,15)$. The flagellar motor consists of a rotor composed by a ring formed by the FliF, FliG, FliM, and FliN proteins that is powered by the proton (or sodium ion) motive force against stators formed by MotA and MotB (or PomA and PomB), which produces torque that is transmitted through the basal body to the hook and, finally, to the filament (15). The rotational motion converts the filament into a screw that propels the water, thus moving the bacterial cell, which, given its small dimensions, moves at a low Reynolds number and therefore lacks inertia (16). The rotational flagellar filament thrusts or drags the cell body, allowing bacteria to swim at speeds on the order of tens of cell body lengths per second.

Among the less-characterized proteins of the flagellar basal body is FliL, which has been investigated in several bacterial species, with most studies carried out in the Gammaproteobacteria species Escherichia coli, Salmonella enterica, Vibrio alginolyticus, and Proteus mirabilis. Meanwhile, research on the Alphaproteobacteria, to which $B$. diazoefficiens belongs, is scarce, and, in particular, there are no reports concerning FliL in the group of $\mathrm{N}_{2}$-fixing rhizobia. This protein was observed by cryo-electron tomography as lying between rotor and stator structures in Borrelia burgdorferi (17). The motor of this spirochete also possesses a particular structure, called the collar, to which FliL is associated (18). Furthermore, biochemical and X-ray crystallography studies in V. alginolyticus suggested that rings of FliL might associate around stator units $(19,20)$. From these and other studies, it may be concluded that FliL is a small membrane protein that is part of the flagellar basal body and that might have a role in stabilizing the stator (21). Hence, when swimming bacteria encounter a surface or when medium viscosity increases, the rotation of the flagellar motor may be impaired, and the flagellar basal body may be subjected to torsional stress. It has been reported that FliL may allow the basal body to withstand this torsional stress by reinforcing the rod, stabilizing the stators and contributing to torque generation (22-25). In E. coli, sensing of the external 
load seems to be performed by a component of the flagellar motor (26), and in $P$. mirabilis the C-terminal portion of FliL was suggested as responsible for sensing the presence of viscosity and surfaces (27-29). However, in E. coli it was reported that, for some minutes, torque generated by the flagellar motors at different loads was independent of FliL (30). Other roles ascribed to FliL are an involvement in the energetics of the flagellar function in $P$. mirabilis (31), the opening of the motor proton channel in Rhodobacter sphaeroides (32), the orientation of periplasmic flagella in B. burgdorferi (17), and the motor rotation in C. crescentus (33).

In addition to the paucity of studies on the role of FliL in alphaproteobacteria, there are no comparative works in bacterial species possessing two flagellar systems except for recent studies in $V$. alginolyticus $(19,20)$. Since $B$. diazoefficiens uses its two flagellar systems for swimming (2), this species brings an opportunity to compare the role of FliL in each system for swimming. Therefore, we addressed this question here by obtaining and characterizing deletion mutants in the fliL genes of each flagellar system in $B$. diazoefficiens. We observed different impacts of the mutations in each fliL paralog and obtained evidence indicating that inactivation of fliL in the subpolar flagellar system modifies the expression of the lateral flagellar master regulator.

\section{RESULTS AND DISCUSSION}

Characterization of FliL $_{\mathbf{s}}$ and FliL $_{\mathbf{L}}$. The open reading frames (ORFs) bll5826 and bll6868 are annotated as fliL in the B. diazoefficiens USDA 110 genomic sequence (34). According to their genomic contexts (see Fig. S1A in the supplemental material), bll5826 encodes the FliL protein of the subpolar flagellar system, while bll6868 would be the FliL of the lateral flagellar system. Therefore, we will refer to these genes as fli $L_{S}$ and $f l i L_{L}$, respectively. fli $L_{S}$ is the first gene of a putative operon consisting of 5 genes related to the subpolar flagellum, while $f_{i} L_{L}$ is localized next to the last gene in operon II of the lateral flagellar system (5). The alignment of $\mathrm{FliL}_{S}$ and $\mathrm{FliL}_{\mathrm{L}}$ amino acid sequences indicated a low relationship, with $19.8 \%$ identity and $40 \%$ similarity between them (Fig. S1B), in agreement with their pertaining to evolutionary different flagellar systems (3). To further characterize their relationship with other FliL proteins, we constructed the cladogram shown in Fig. 1, and we found that FliL distribution was similar to that previously described for other flagellar proteins (2). In particular, FliL $_{\mathrm{s}}$ and $\mathrm{FliL}_{\mathrm{L}}$ were grouped in separate clades, where $\mathrm{FliL}_{\mathrm{S}}$ was related to homologs from $C$. crescentus and the primary system of Rhodopseudomonas palustris, while $\mathrm{FliL}_{\mathrm{L}}$ grouped with those of Tardiphaga sp. and the secondary system of $R$. palustris. Moreover, FliL from E. meliloti, Bacillus subtilis, B. burgdorferi, and the secondary FliL from $R$. sphaeroides were more related to $\mathrm{FliL}_{\mathrm{L}}$ than to $\mathrm{FliL}_{\mathrm{S}}$, whereas FliL from gammaproteobacteria and the primary system of $R$. sphaeroides were unrelated to both $B$. diazoefficiens FliL. Interestingly, $B$. diazoefficiens Fli $_{s}$ tightly grouped in the same clade with those FliL of the subpolar flagellum from Bradyrhizobium species that possess two flagellar systems, while FliL from species like Bradyrhizobium elkanii, which possess a single (subpolar) flagellar system, were grouped in a separate clade (Fig. 1). In V. alginolyticus, FliL appears to be in contact with the MotB (PomB) of the stator complex in the periplasmic space (19), whereby some complementarity between the structural domains of both proteins is expected. Hence, the above results may indicate some structural difference between subpolar flagellar stators in the groups of Bradyrhizobium species that possess single or dual flagellar systems.

The domain prediction of these polypeptides indicated that, as described for other FliL proteins, both homologs have a single transmembrane domain. For FliL $_{s}$, most of the algorithms employed predict the $23 \mathrm{~N}$-terminal residues as a cytoplasmic domain followed by a 20-residue transmembrane domain and then predict a 123-residue $\mathrm{C}$-terminal domain within the periplasm. Intriguingly, the consensus prediction for $\mathrm{FliL}_{\mathrm{L}}$ is $3 \mathrm{~N}$-terminal residues at the periplasm followed by the 20-residue transmembrane domain and then a 125-residue C-terminal domain in the cytoplasm (Fig. S1D). However, the probability of the C-terminal domain being cytoplasmic is only slightly higher than that of it being periplasmic. This domain distribution does not agree with previous 


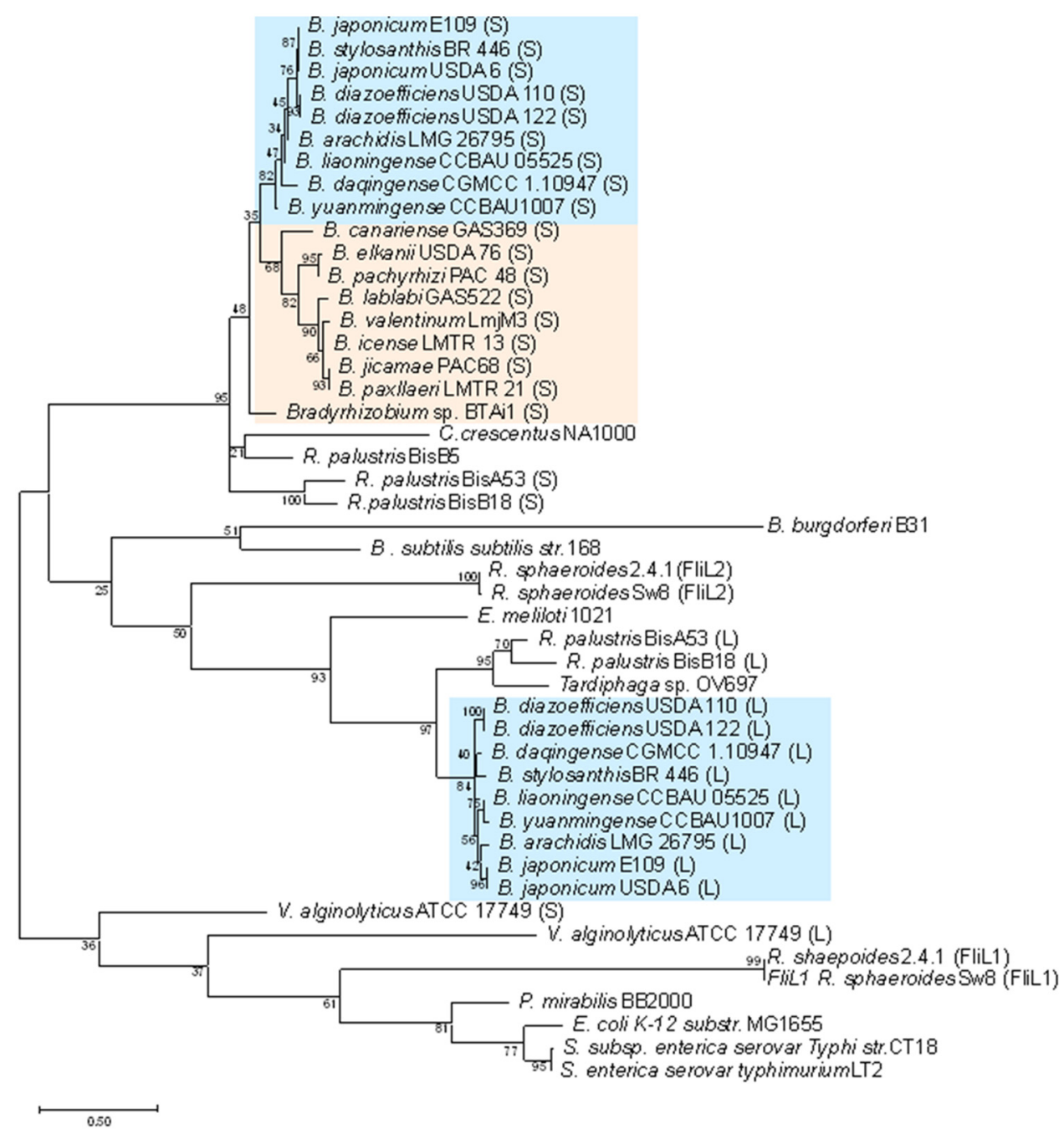

FIG 1 Maximum-likelihood cladogram of FliL from Bradyrhizobium spp. and related species. In cases where two flagellar systems were identified in the genome sequence, FliL paralogs similar to the $B$. diazoefficiens USDA 110 subpolar system were indicated as $S$, and those similar to the $B$. diazoefficiens USDA 110 lateral system were indicated as L. In species where two flagellar systems were identified but their genomic sequences did not allow their assignations as subpolar or lateral, the FliL paralogs were indicated as FliL1 or FliL2. Blue rectangles highlight strains belonging to the $B$. japonicum phylogroup 1 , while the pink rectangle highlights strains from other Bradyrhizobium spp. phylogroups, as described elsewhere (3). Next to the nodes, the percentages of replicate trees in which the associated taxa clustered together in the bootstrap test with 1,000 replicates are shown. Branch lengths in the tree are scaled in the same units as those of the evolutionary distances (scale bar) used to infer the phylogenetic tree.

studies of FliL and in particular with the proposed interaction of FliL with the stator complex in the periplasmic side (24). Therefore, we will not assume this domain disposition for Fli $\mathrm{L}_{\mathrm{L}}$ until experimental confirmation.

In searching for additional evidence supporting the roles of $\mathrm{FliL}_{S}$ and $\mathrm{FliL}_{L}$, we intended to complement a well-known FliL defect. In enteric bacteria such as E. coli and Salmonella spp., inactivation of FliL leads to a small defect in swimming but produces a substantial reduction in swarming (23). To see whether the B. diazoefficiens fliL paralogs are able to complement this mutation in E. coli, we cloned the wild-type (WT) fli $_{S}$ and fli $L_{L}$ genes from B. diazoefficiens USDA 110 into the pBAD24 vector and transformed an E. coli MG1655 $\Delta$ fliL mutant with these plasmids, along with the empty vector as a negative control. In agreement with previous reports (23), swimming was marginally affected in the MG1655 $\Delta$ fliL mutant, while swarming was strongly reduced. $B$. diazoefficiens fli $L_{L}$ partially complemented these defects in the MG1655 $\Delta$ fliL mutant 
A HMY-arabinose

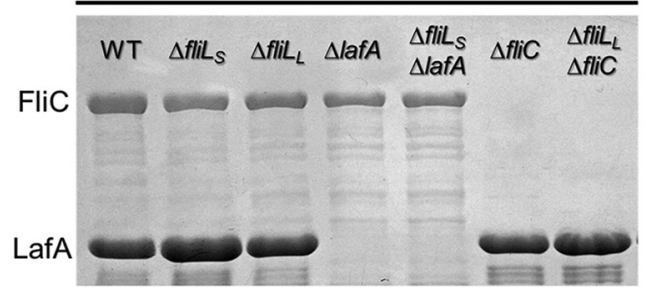

B

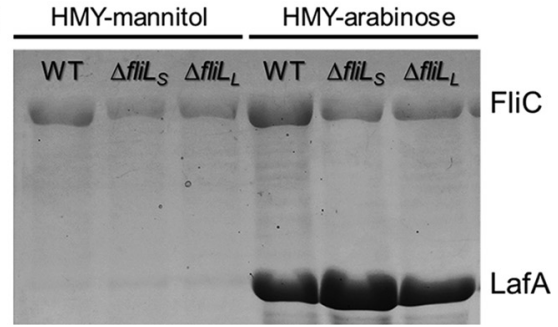

FIG 2 Extracellular proteins from B. diazoefficiens USDA 110 and their fliL mutant relatives in liquid medium. (A) $B$. diazoefficiens wild-type (WT), $\Delta$ fli $L_{S}, \Delta$ fliL $L_{L}, \Delta$ lafA, $\Delta$ fliC, $\Delta$ fliL $L_{S} \Delta l a f A$, and $\Delta$ fliL $L_{L} \Delta$ fliC strains grown in liquid $H M Y$ medium with L-arabinose as the carbon source. (B) B. diazoefficiens WT, $\Delta$ fli $_{S}$, and $\Delta$ fliL $L_{L}$ strains grown in liquid $H M Y$ medium with D-mannitol or L-arabinose as the carbon source. The extracellular proteins were separated by SDS-PAGE and stained with Coomassie brilliant blue. The positions of the flagellins FliC and LafA (subpolar and lateral, respectively) are indicated at the sides of the images.

(Fig. S2), indicating that, despite its low sequence relatedness and atypical domains prediction, Fli $L_{L}$ may be considered a functional FliL protein. However, $B$. diazoefficiens $\mathrm{fliL}_{\mathrm{s}}$ did not complement the E. coli $\Delta$ fliL mutation (Fig. S2). To seek an explanation of this difference in complementation, we compared the $B$. diazoefficiens subpolar and lateral stator sequences with that of $E$. coli. There are mot $A B$ and pom $A B$ stator genes annotated in the clusters of the $B$. diazoefficiens subpolar flagellar system, but whether one or both classes of stators are used by this bacterium is unknown. Therefore, we compared both sets of stator proteins with $E$. coli Mot $A B$ and found that Mot $A B$ and Pom $A B$ of the subpolar system were unrelated to $E$. coli MotAB (Fig. S3). In contrast, the ORFs annotated as MotA and MotB in the lateral flagellar genes cluster were related to those of E. coli MotAB, in the same branch as those of Aeromonas hydrophila and Pseudomonas aeruginosa (Fig. S3). Thus, the lack of complementation of the E. coli mutation by the wild-type fli $L_{s}$ might be due to insufficient fit between Fli $_{s}$ and the $E$. coli stators, whereas Fli $\mathrm{L}_{\mathrm{L}}$ might adjust better. Therefore, these results are not enough to rule out FliL functionality of Fli $\mathrm{L}_{\mathrm{S}}$ in $B$. diazoefficiens.

Swimming speed was differentially affected by mutations in $\mathrm{fli}_{S}$ or $\mathrm{fli}_{L}$. We obtained deletion mutants in each $B$. diazoefficiens fliL paralog by removing internal fragments from their coding sequences (Fig. S1 A and C). Since both genes were located in operons, we performed unmarked in-frame deletion mutants to avoid possible polar effects. We observed that the $\Delta$ fli $L_{S}$ and $\Delta f l i L_{L}$ mutants produced the same subpolar and lateral flagellins as the wild type, indicating that they possessed both types of flagella; however, the level of LafA relative to FliC was higher in the $\Delta$ fliL $L_{s}$ mutant (Fig. 2). In mutants possessing only one flagellar system, neither fliL deletion provoked changes in the remaining flagellin (Fig. 2A). In addition, both mutants responded to the carbon source as previously observed $(5,11,12)$; the lateral flagellins were not produced by bacteria grown in HM salts-yeast extract (HMY) with D-mannitol as a carbon source and were produced by bacteria grown in HMY with L-arabinose (Fig. 2B).

Swimming speed in liquid HMY medium with L-arabinose was affected by these mutations, although the effects were different for each single-mutant strain. As shown in Table 1, we corroborated in the background of USDA 110 the swimming speed values previously reported for the LP 3004 wild type and two flagellin mutants, a $\Delta$ lafA mutant, lacking the lateral flagellins, and a $\Delta$ fliC mutant, lacking the subpolar flagellins (2). In addition, deletion in fli $L_{S}$ led to only a $29 \%$ reduction in swimming speed in both a $\Delta f i_{s}$ single mutant possessing both flagella and a $\Delta f L_{s} \Delta$ lafA double mutant unable to produce lateral flagellar filaments, indicating that in $B$. diazoefficiens Fli $_{s}$ is not essential for subpolar flagellar function in swimming. These results are in contrast to previous observations in C. crescentus-an alphaproteobacterium that has a flagellar system closely related to the subpolar flagellar system of $B$. diazoefficiens-in which FliL was reported as essential for flagellar motor rotation (33).

The deletion in $f_{i l} L_{L}$ did not produce a motility reduction in cells possessing both flagella; however, when $\Delta f L_{L}$ was combined with a $\Delta$ flic mutation, (i.e., lacking 
TABLE 1 Swimming speed of $B$. diazoefficiens wild-type and mutant strains in HMYarabinose medium $^{a}$

\begin{tabular}{lll}
\hline Strain description & Speed $\left(\mu \mathrm{m} \cdot \mathrm{s}^{-1}\right) \pm \mathrm{SD}^{c}$ & $n$ \\
\hline WT & $27.59 \pm 0.54 \mathrm{~A}$ & 160 \\
$\Delta$ lafA & $30.44 \pm 0.67 \mathrm{~A}$ & 118 \\
$\Delta$ fliC & $17.21 \pm 0.25 \mathrm{~B}$ & 77 \\
$\Delta$ fliL & $19.63 \pm 0.91 \mathrm{~B}$ & 91 \\
$\Delta$ fliL $_{S} \Delta$ lafA & $21.65 \pm 0.37 \mathrm{~B}$ & 120 \\
$\Delta$ fliL & $28.65 \pm 0.26 \mathrm{~A}$ & 104 \\
$\Delta$ fliL $_{L} \Delta$ flic & $0^{b}$ & \\
\hline
\end{tabular}

${ }^{a}$ Movies of swimming cells were recorded at $30 \mathrm{frames} / \mathrm{s}$ and the speed was measured using Move-tr/2D software.

${ }^{b}$ No data are presented for the $\Delta$ fli $_{L} \Delta$ fliC strain because this mutant was nonmotile.

The data represent the mean speed of $n$ swimming cells \pm standard deviation (SD). Values followed by different letters were statistically different according to analysis of variance (ANOVA) followed by Tukey's multiple-comparison test with $\alpha=0.005$.

subpolar flagellar filaments), cells were nonmotile (Table 1). These results indicate a moderate requirement of FliL $\mathrm{L}_{\mathrm{s}}$ for swimming with subpolar flagellum and an essential role of Fli $L_{L}$ for swimming with lateral flagella.

In E. coli, S. enterica, P. mirabilis, and V. alginolyticus, FliL was reported as required for swimming in viscous environments, including swarming behavior, which might be related to a role for this protein in flagellar stabilization $(21-24,27)$. Therefore, we investigated the effects of deletions in both fliL paralogs when medium viscosity was increased by the addition of polyvinylpyrrolidone (PVP). At a low PVP concentration (1\% wt/vol), swimming speeds of $\Delta$ fli $_{s}$ (with both flagella) and $\triangle$ fli $L_{s} \Delta$ lafA (with only subpolar flagellum) mutants were only slightly reduced compared to those in the medium without PVP. When PVP concentrations rose to $2 \% \mathrm{wt} / \mathrm{vol}$ or more, swimming speeds decreased steadily in all strains, showing again the difficulty of the subpolar flagellum in propelling the cells in a viscous environment (Fig. $3 \mathrm{~A}$ ). However, $\Delta$ fli $L_{L}$ mutant swimming speed was not reduced and remained comparable to that of the wild type at low PVP concentration (1\% wt/vol) and was significantly reduced with PVP above $2 \% \mathrm{wt} / \mathrm{vol}$ (Fig. 3B), showing the requirement of this protein by the lateral flagella in viscous media.
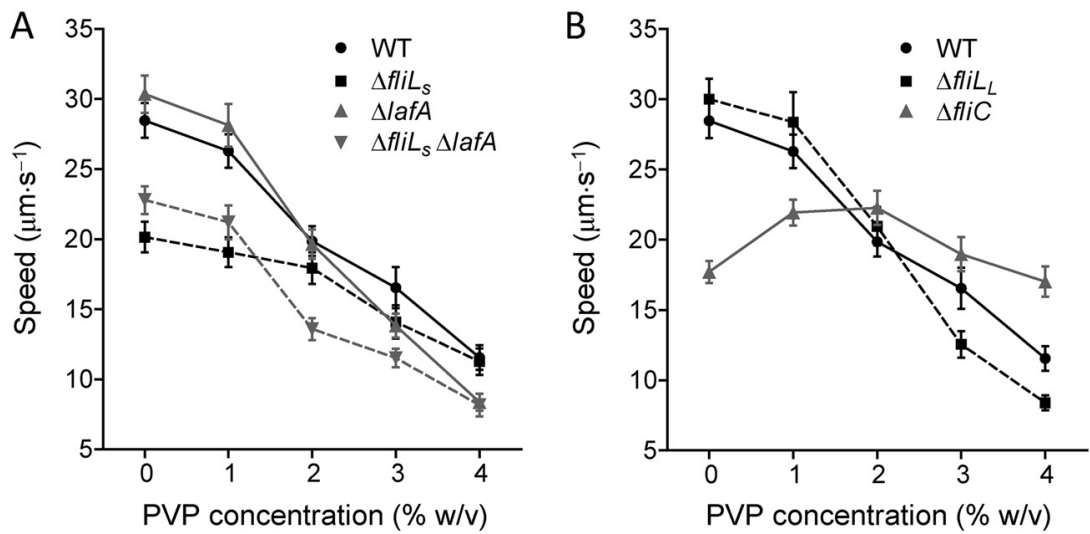

FIG 3 Effect of viscosity on B. diazoefficiens swimming speed. Cells were grown in HMY-arabinose medium and diluted in HM salts containing different concentrations of polyvinylpyrrolidone (PVP). Swimming speeds of at least 60 cells from two biological replicates were recorded with Move-tr/2D. Data points are mean $\pm 99 \%$ confidence intervals calculated with Student's $t$ test. (A) Comparison of $\Delta$ fli $L_{s}$ against wild-type $\mathrm{fliL}_{S}$ in the background of the wild-type cells that possess both flagellar systems or in the background of the $\triangle$ lafA mutant, which lacks lateral flagellar filaments. (B) Comparison of $\Delta$ fli $L_{L}$ against wild-type fli $L_{L}$ in the background of the wild-type cells that possess both flagellar systems or in the background of the $\Delta$ fliC mutant, which lacks subpolar flagellar filaments. The $\Delta f l i L_{\perp} \Delta$ flic double mutant did not swim under any condition assayed. All the strains were assayed at the same time and presented in separate charts for clarity. 


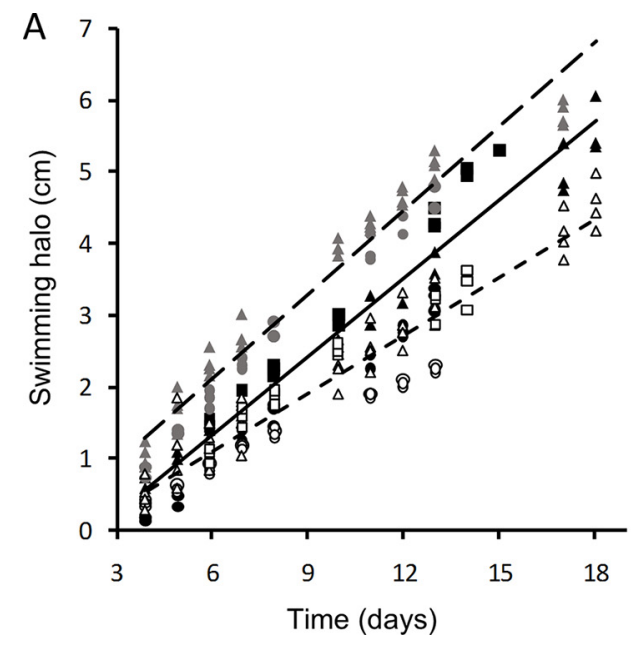

\section{B}

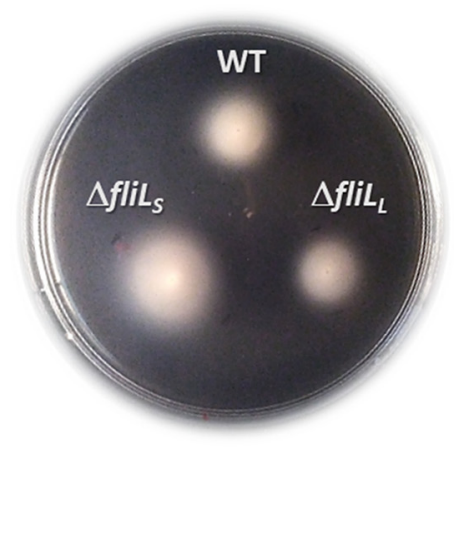

FIG 4 Swimming motility of $B$. diazoefficiens USDA 110 in semisolid Götz minimal medium with D-mannitol as the carbon source. Cells were inoculated at the center of $0.3 \%$ (wt/vol) agar plates and incubated at $28^{\circ} \mathrm{C}$ for the indicated times. (A) Diameter of the swimming halo of wild-type (WT; black symbols), $\Delta$ fli $L_{S}$ (gray symbols), and $\Delta f l i L_{L}$ (white symbols) strains. The complete data set from two ( $\Delta$ fli $L_{S}$ strain) or three (WT and $\Delta$ fli $L_{L}$ strains) independent experiments with three or four technical replicates is shown. Each experiment is distinguished by a different symbol. Regression lines for each set are as follows: WT, continuous line $\left(r^{2}=0.89\right) ; \Delta$ fli $L_{S}$ strain, long-dashed line $\left(r^{2}=0.95\right)$; and $\Delta f l i L_{L}$ strain, short-dashed line $\left(r^{2}=0.90\right)$. (B) Representative $90-\mathrm{mm}$ plate after 8 days of incubation.

Swimming in semisolid medium. Swimming behavior may be evaluated in semisolid medium containing $0.3 \%$ agar in which bacteria have to swim while migrating through the agar mesh (35). In this environment, $B$. diazoefficiens produces both subpolar and lateral flagella, even when D-mannitol is the only carbon source (2). We studied swimming motility in Götz-mannitol semisolid medium, which is a minimal medium developed for studies of motility in rhizobia and is well suited for this kind of study with $B$. diazoefficiens $(9,36)$. Although the $\Delta f l L_{L}$ mutant was less motile, $\Delta f l i L_{S}$ motility haloes were more expanded than those of the wild type (Fig. 4). When we added PVP at two concentrations to obtain middle ( $2 \% \mathrm{wt} / \mathrm{vol})$ and high ( $5 \% \mathrm{wt} / \mathrm{vol})$ viscosity, we observed contrasting effects of each fliL mutation (Fig. 5). In agreement with the changes in swimming speed, the motility haloes of the $\Delta f \mathrm{fl}_{s}$ mutant in the background of the wild type (with both flagella) decreased when PVP increased at $2 \%$
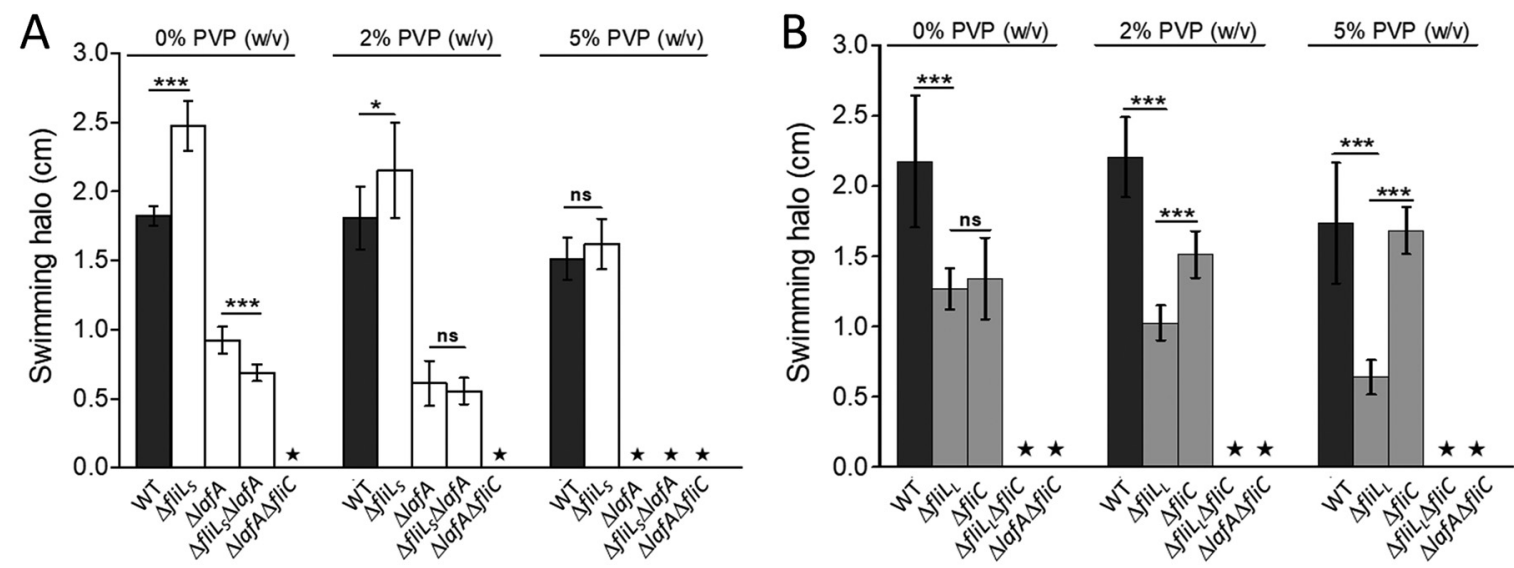

FIG 5 Swimming motility of $B$. diazoefficiens USDA 110 and its mutant relatives in semisolid Götz minimal medium with D-mannitol as the carbon source, supplemented with polyvinylpyrrolidone (PVP). (A) Effects of the $\Delta f_{l i L_{s}}$ deletion. (B) Effects of the $\Delta$ fliL $L_{L}$ deletion. The nonmotile $\triangle$ lafA $\triangle$ fliC mutant, lacking all flagellins, was included as negative control. The bacteria were inoculated on Götz-mannitol soft agar $(0.3 \% \mathrm{wt} / \mathrm{vol})$ with the indicated PVP concentrations and incubated at $28^{\circ} \mathrm{C}$ for 8 days. Averages \pm SD are plotted. Data are representative of two biological replicates, with five technical replicates per experiment. Significant differences were estimated by analysis of variance (ANOVA); ${ }^{*}, \alpha=0.05 ;{ }^{* * *}, \alpha=0.001 ;$ ns, nonsignificant. Stars indicate absence of movement. 

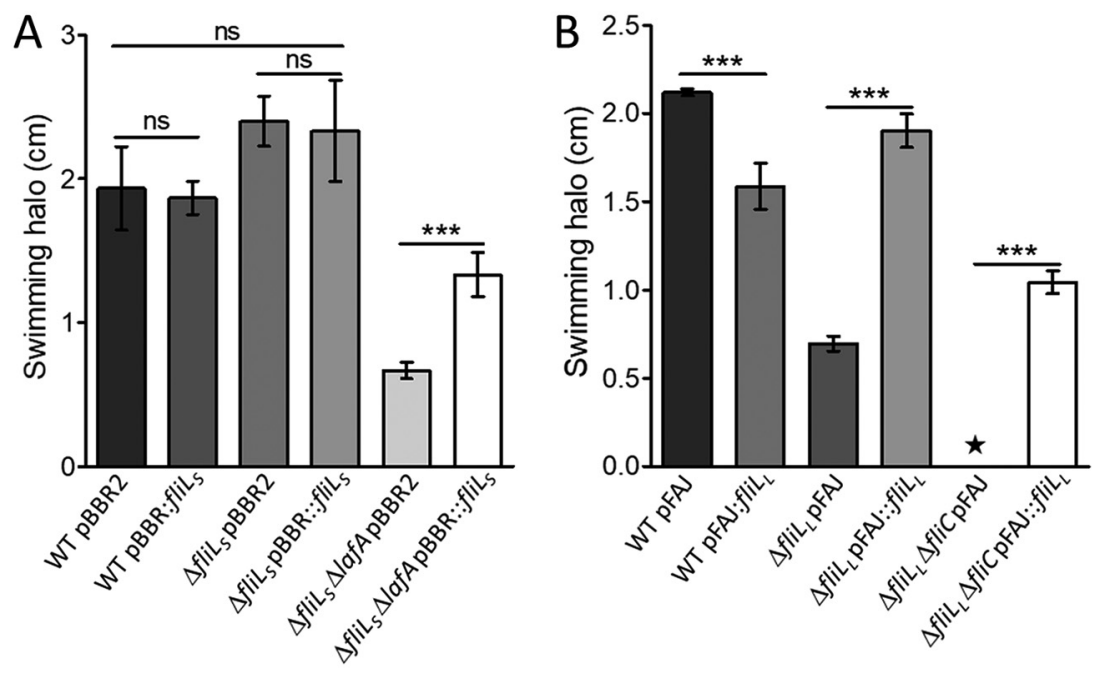

FIG 6 Complementation of fliL mutations in B. diazoefficiens USDA 110 for swimming motility in semisolid Götz minimal medium with D-mannitol as the sole carbon source. (A) Complementation of $\Delta$ fli $L_{s}$ by the wild-type fli $_{S}$ carried in the replicative vector pBBR1MCS2 (pBBR::fli $L_{s}$ ) in $0.3 \%$ (wt/vol) agar. (B) Complementation of $\Delta$ fliL by the wild-type fliL carried in the replicative vector pFAJ1708 (pFAJ::fliL $)$ in $0.3 \%$ (wt/vol) agar with the addition of 5\% (wt/vol) PVP. The empty vectors, abbreviated pBBR2 and pFAJ, respectively, were used as controls. Swimming motility was quantified by the diameter of haloes produced 8 days after inoculation at $28^{\circ} \mathrm{C}$. Data are averages from three biological replicates with 6 technical replicates. Statistical analysis was carried out by ANOVA. ${ }^{* *}, \alpha=0.001$; ns, nonsignificant. The star indicates absence of movement.

and $5 \%$ (wt/vol). In turn, the $\triangle f_{l i L_{s}} \triangle l a f A$ mutant (with only subpolar flagellum) produced motility haloes of reduced size in semisolid medium with $2 \%$ (wt/vol) PVP and did not move in semisolid medium with 5\% (wt/vol) PVP (Fig. 5A). Moreover, motilities of the $\triangle f_{l i L_{s}} \Delta l a f A$ double mutant and the $\Delta$ lafA single mutant were similar in viscous medium ( $2 \%$ and $5 \%$ PVP), indicating that the lack of lateral flagellins was epistatic on the fli $L_{S}$ mutation. Regarding the $\Delta f l i L_{L}$ strain, we observed that its motility diminished with the increase in viscosity in the wild-type background, but the $\Delta f L_{L}$ $\triangle$ fliC double mutant was nonmotile under all conditions (Fig. 5B), indicating an essential role of $\mathrm{FliL}_{\mathrm{L}}$ for the activity of lateral flagella in viscous semisolid medium. In agreement with previous results (2), the motility of the $\Delta$ fliC single mutant, which has only lateral flagella, was unaffected by viscosity.

Complementation of fliL mutants. To assess whether fli $L_{S}$ and fli $L_{L}$ deletions may be complemented in trans by the wild-type alleles, both full-length fliL sequences were cloned in replicative vectors and introduced into each strain, with empty vectors used as controls. We performed complementation experiments in semisolid medium under the conditions where each mutant showed the most differential phenotype, namely, Götz-mannitol for the $\Delta f_{\text {fil }}$ mutant and the same medium supplemented with $5 \%$ PVP for the $\Delta f \mathrm{fl}_{L}$ mutant. The results of complementation experiments are shown in Fig. 6 .

$\Delta$ fli $L_{S}$ mutants in the background that possessed both flagellar systems produced haloes slightly wider than those of the wild type carrying either empty pBBR1MCS2 (referred to as pBBR2 in Fig. 6) or pBBR2::fliL $L_{S}$ albeit without statistically significant differences (Fig. 6A, left four bars). These effects might be related to some instability caused by overexpression of the proteins from the vector. In addition, the in-frame fli $L_{S}$ deletion generated for this study left a coding sequence remnant that might produce a peptide of 39 amino acid (aa) residues. This putative peptide possesses an 8-aa stretch at its $C$ terminus that might interfere at least partially with proper association of $\mathrm{FliL}_{\mathrm{S}}$ subunits translated from pBBR2::fliL $L_{s}$ in unbalanced stoichiometry (Fig. S1C). Such interference might preclude the expected suppression of increased lateral flagellummediated swimming. In bacteria lacking lateral flagella, the partial loss of motility of the $\Delta f l i L_{s} \Delta l a f A$ double mutant with respect to the $\Delta l a f A$ mutant was suppressed by the 
full-length fli $_{S}$ introduced in pBBR2::fliL $L_{S}$ in comparison with the isogenic $\triangle$ fli $L_{S} \Delta l a f A$ double mutant carrying the empty vector (Fig. 6A). Since this double mutant was constructed by replacing lafA12 with $\triangle$ lafA into the same $\triangle f l i L_{S}$ background used above, successful complementation in the $\triangle$ lafA background suggests that lack of differences in the WT background was not due to a polar effect of the $\Delta f_{i l} L_{s}$ mutation.

Regarding the lateral flagellar system, ectopic expression of fli $L_{L}$ in the wild-type background somewhat diminished swimming motility with respect to the wild type carrying empty vector. Nevertheless, the low-motility phenotype of the $\Delta f L_{L}$ mutant was completely reverted by the wild-type allele (Fig. 6B). As observed before, the $\Delta f i L_{L}$ $\triangle$ fliC double mutant, which does not possess functional subpolar flagella, was nonmotile. Introduction of the wild-type copy of fli $L_{L}$ restored motility to this strain, indicating that lack of motility of the $\Delta f L_{L} \Delta f$ fliC double mutant was due solely to $\Delta f l i L_{L}$ deletion.

Lateral flagellar gene expression increased in the $\Delta$ fli $_{s}$ mutant. Given the role of lateral flagella for swimming in semisolid agar and in viscous medium, we considered the possibility that dysfunction of $f_{i l} L_{s}$ provokes an induction of the lateral flagellar system expression. In other species, it is known that the flagella may act as a mechanosensor, and the C-terminal domain of FliL was implicated as responsible for sensing the presence of surfaces to trigger differentiation into swarmer phenotypes (27). In addition, it was observed in Vibrio parahaemolyticus that mutation in the gene of the polar flagellin fliC provoked differentiation of planktonic cells into elongated, hyperflagellated swarmer cells in liquid medium, where these kinds of cells or changes do not otherwise occur (37). To observe if there is an induction of lateral flagellar synthesis, we performed retrotranscribed quantitative PCR (RT-qPCR) with primers directed at the class IB lateral flagellum master regulator lafR (5) with RNA obtained from wild-type and $\Delta f_{i l} L_{S}$ cells grown in liquid peptone-salts yeast extract (PSY)-arabinose (Ara) medium. There was a significant increase in lafR transcript in the $\triangle f_{l i L_{s}}$ mutant (Fig. 7A), and concomitantly, the mutant cells produced more LafA flagellins in both HMY-Ara and PSY-Ara media (Fig. 2 and 7B). We also extracted RNA from swimming haloes in semisolid PSY-Ara medium. We took cells from the whole motility halo, as well as from a region confined to the $1.5-\mathrm{cm}$ exterior border of the halo, and compared the transcript amounts of lafR. We found no significant differences between the wild type and the mutant when RNAs were obtained from the whole motility halo, but when they were from the exterior border, lafR was significantly overexpressed in the $\Delta f L_{S}$ mutant (Fig. 7C). We also measured transcript accumulation of flif $F_{L}, \operatorname{mot} C$, and fliL $L$ in the exterior border of the motility halo, because these genes are representatives of two different laf operons (5). As shown in Fig. 7D, all of these genes were overexpressed in the $\Delta f i L_{S}$ mutant with respect to the wild type, thus indicating that fli $L_{s}$ absence induced an overproduction of lateral flagellum-related genes. In agreement with these results, the $\Delta$ fli $_{s}$ mutant cells produced many more lateral flagella at the border of the motility halo $(\mathrm{BMH})$ than did the wild-type cells at this same position (Fig. 8). However, there were no significant differences in motility halo diameters between the wild type and the $\Delta$ fliL $L_{S}$ mutant in PSY-Ara semisolid medium. This result is expected, since it is known that swimming motility is less stimulated in rich media $(35,38)$. Since we could not obtain good RNA samples from swimming haloes in semisolid Götz minimal medium, we decided to corroborate the higher lateral flagellum production by the mutant in this medium by directly observing (via transmission electron microscopy) the number of lateral flagella. As shown in Fig. 8, we confirmed that the $\Delta f^{\prime i} L_{s}$ mutant produced more lateral flagella than did the wild type at the border of motility haloes in both PSY-Ara and Götz-mannitol media.

Taken together, these results suggest that either lack of fli $L_{S}$ or subpolar flagellum destabilization was a signal for lafR transcript accumulation. Therefore, there may be some cross talk between both flagellar systems that is able to regulate the production of the lateral flagella in response to the activity/stability of the subpolar flagella. This supposed cross talk should be unidirectional from the subpolar to the lateral system, 
A
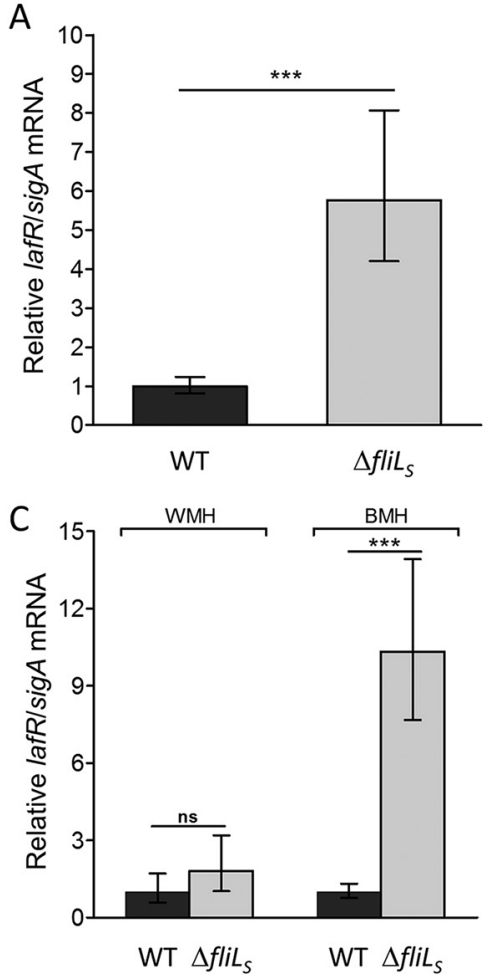

B

Flic
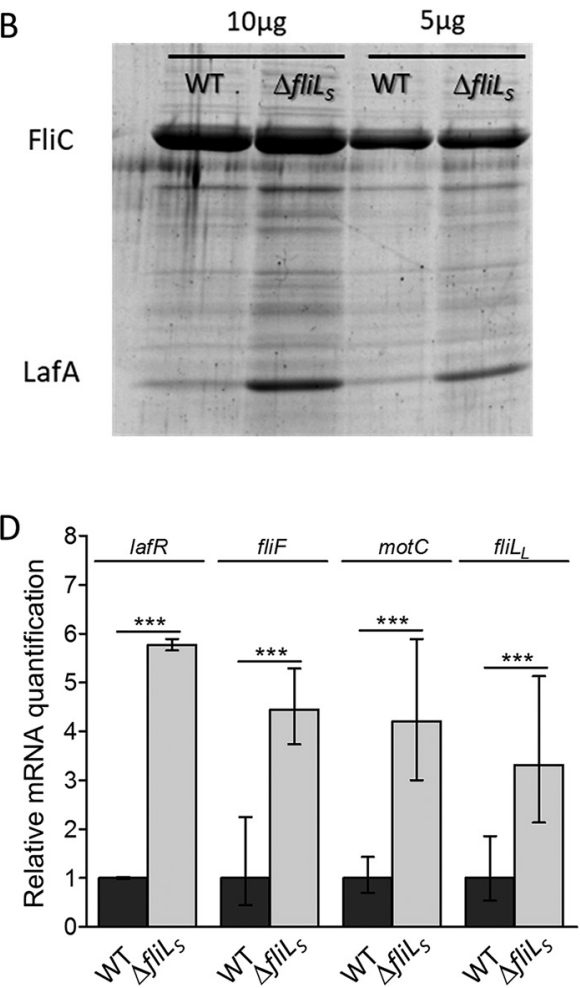

FIG 7 Expression of lateral flagella in the wild type and $\triangle$ fliL $_{S}$ mutant in PSY-arabinose. (A) lafR transcript quantification by retrotranscribed quantitative PCR (RT-qPCR) with respect to the sigA transcript as the constitutive control, with mRNA extracted from liquid cultures. (B) FliC and LafA flagellins extracted from liquid cultures. The amounts of proteins loaded in the SDS-PAGE are indicated above the lanes. (C) mRNA extracted from the border of a motility halo $(B M H)$ or from the whole motility halo (WMH). (D) Transcript quantification by RT-qPCR of LafR target genes from BMH with respect to the sigA transcript as constitutive control. Data presented in panels $A$ and $B$ are the averages \pm SD from three and two biological replicates, respectively, each with three technical replicates. Data in panel D are representative from two biological replicates. Statistical analyses were carried out by ANOVA. ${ }^{* *}, \alpha=0.001 ; \mathrm{ns}$, nonsignificant.

since no effects on FliC synthesis or function could be observed as consequence of fli $L_{L}$ alteration.

Conclusions. Our results indicate that, in contrast to previous reports in C. crescentus, FliL $_{\mathrm{s}}$ is not essential for subpolar flagellar function in swimming but might play a role in the control of lateral flagellum synthesis both in liquid medium and in response to an increase in viscosity. Under these conditions, Fli $_{\mathrm{L}}$ seemingly performs a critical role in stabilizing lateral flagella for swimming.

The possession of two flagellar systems, however, is not a general trait of the Bradyrhizobium genus (3). B. elkanii is another important soybean symbiont that possesses only one flagellar system, similar to the $B$. diazoefficiens subpolar system. Furthermore, the type strain, $B$. elkanii USDA 76, moved equally well as $B$. diazoefficiens USDA 110 in semisolid medium without the addition of a viscous agent (2), and $B$. elkanii soil isolates were even more motile than $B$. diazoefficiens or Bradyrhizobium japonicum under these conditions (39), indicating that the single flagellar system of $B$. elkanii was enough for efficient swimming in such a tortuous environment. However, $B$. elkanii was unable to swim in viscous medium (2). Since the lateral flagellar system consumes a considerable proportion of metabolic energy (12) and, as inferred from phylogenetic analyses, it was acquired at the separation of the B. japonicum phylogroup from the other Bradyrhizobium phylogroups (3), we speculate that the main lateral flagellar function is allowing swimming in viscous medium, which should be a distinctive trait of the $B$. japonicum phylogroup, although it is not essential for swimming in general. The viscosity of soil solution might increase as a consequence of desiccation, 
WT

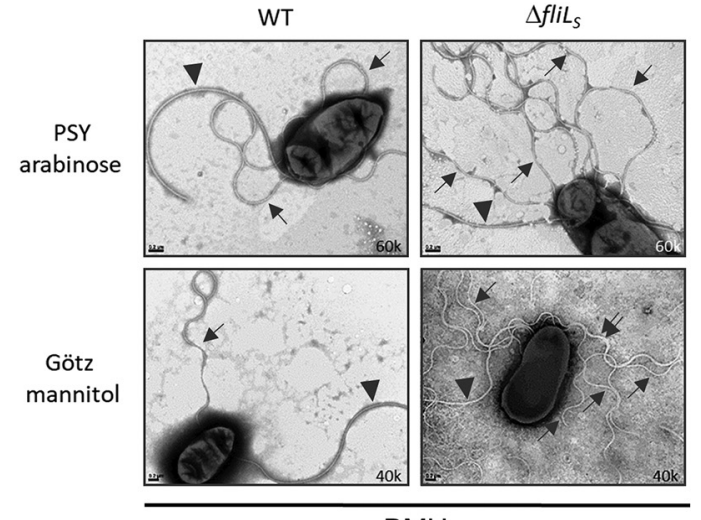

$\mathrm{BMH}$
WT
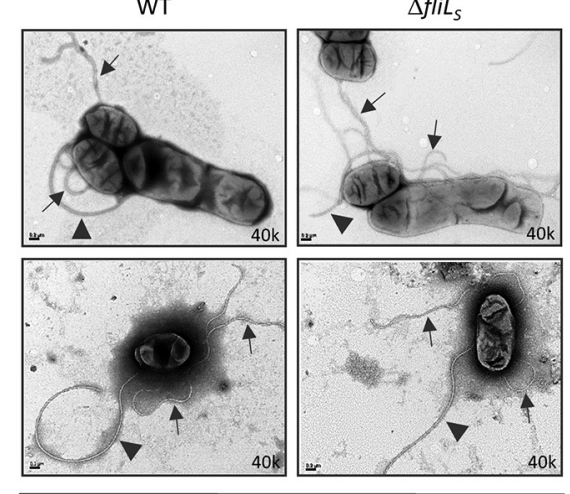

WMH

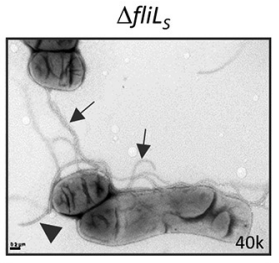

FIG 8 Transmission electron micrographs of individual $B$. diazoefficiens wild-type and $\Delta$ fliL $L_{s}$ mutant cells obtained from the border of the motility halo $(\mathrm{BMH})$ or from the whole motility halo (WMH) from semisolid (agar $0.3 \%)$ PSY-arabinose (top) and Götz-mannitol (bottom). WT cells in BMH possessed a subpolar flagellum (arrowhead) and one or two lateral flagella (arrows), while the $\Delta f l i L_{s}$ mutant exhibited a subpolar flagellum and more than two lateral flagella per cell, some of which are partially bundled (double arrow). In contrast, in WMH the flagellar patterns were similar between WT and mutant strains in both media. Bars, $0.2 \mu \mathrm{m}$. 40k, $\times 40$ magnification; $60 \mathrm{k}$, $\times 60$ magnification.

when the concentration of solutes and colloids increase. Therefore, we hypothesize that the B. japonicum phylogroup might have evolved adaptations to drier soil environments, where the lateral flagella might play an essential role for motility under such conditions. For this adaptation, each FliL seems to play a distinct and specific role.

\section{MATERIALS AND METHODS}

Bacterial strains and culture conditions. Strains and plasmids used in this study are listed in Table S1 in the supplemental material. B. diazoefficiens cells were stored in glycerol stocks at $-20^{\circ} \mathrm{C}$ and, for routine use, in yeast extract-mannitol agar (YMA) (40) at $4^{\circ} \mathrm{C}$. For conjugation, PSY-arabinose was used (41). If needed, chloramphenicol $(\mathrm{Cm})$, tetracycline $(\mathrm{Tc})$, kanamycin $(\mathrm{Km})$, gentamicin $(\mathrm{Gm})$, streptomycin $(\mathrm{Sm})$, or spectinomycin (Sp) was added at a final concentration of $20 \mu \mathrm{g} \cdot \mathrm{ml}^{-1}, 50 \mu \mathrm{g} \cdot \mathrm{ml}^{-1}, 150 \mu \mathrm{g} \cdot \mathrm{ml}^{-1}, 100 \mu \mathrm{g} \cdot \mathrm{ml}^{-1}$, $400 \mu \mathrm{g} \cdot \mathrm{ml}^{-1}$, or $200 \mu \mathrm{g} \cdot \mathrm{ml}^{-1}$, respectively. E. coli strains were grown at $30^{\circ} \mathrm{C}$ in LB (42). If needed, tetracycline $(\mathrm{Tc})$, kanamycin $(\mathrm{Km})$, gentamicin $(\mathrm{Gm})$, streptomycin $(\mathrm{Sm})$, spectinomycin $(\mathrm{Sp})$, or ampicillin (Ap) was added at a final concentration of $10 \mu \mathrm{g} \cdot \mathrm{ml}^{-1}, 25 \mu \mathrm{g} \cdot \mathrm{ml}^{-1}, 10 \mu \mathrm{g} \cdot \mathrm{ml}^{-1}, 100 \mu \mathrm{g} \cdot \mathrm{ml}^{-1}$, or $200 \mu \mathrm{g} \cdot \mathrm{ml}^{-1}$, respectively.

To visualize both kinds of flagella in liquid medium, as well as to measure swimming speed, bacteria were cultured in HMY medium $(5,43)$ supplemented with L-arabinose; for experiments in semisolid medium, minimal Götz medium (36) was used; to obtain high-quality RNA, PSY medium (41) supplemented with L-arabinose was used. The comparison of the three media compositions is shown in Table S2. Mutations studied in this paper did not affect growth kinetics in any of these media (Fig. S4).

Bioinformatic methods. Sequences of the putative FliL polypeptides for the subpolar flagellum (FliL $L_{s}$ ) and the lateral flagellum ( $\mathrm{FliL}_{\mathrm{L}}$ ) were obtained from the MicrobesOnline server (44), while sequences used for phylogenetic analysis were recovered from http://img.jgi.doe.gov or https://www .ncbi.nlm.nih.gov. Protein secondary structures were predicted using Topcons (45) and TMHMM (46). Multiple sequence alignments were performed using ClustalW and MegaX software (47). The evolutionary history was inferred by using the maximum likelihood method and the Le_Gascuel_2008 model (48). Initial trees for the heuristic search were obtained automatically by applying the neighbor-joining and BioNJ algorithms to a matrix of pairwise distances estimated using the Jones-Taylor-Thornton (JTT) model, and then selecting the topology with the superior log likelihood value. A discrete gamma distribution was used to model evolutionary rate differences among sites (5 categories [+ $G$, parameter $=6.6557])$. The tree was drawn to scale, with branch lengths measured in the number of substitutions per site.

Construction of mutant and complemented strains. The general cloning procedures were performed as described (42). Biparental matings with B. diazoefficiens were performed with E. coli S17-1, and electroporation was performed using a GenePulser instrument as previously reported (5).

DNA amplifications were carried out by PCR using Pfu polymerase (Productos Bio-Logicos, Buenos Aires, Argentina) or Taq DNA polymerase (Productos Bio-Logicos, Buenos Aires, Argentina) in a Biometra TOne thermocycler (Analytik Jena, Jena, Germany). The oligonucleotide primers used in this study were supplied by Genbiotech SRL (Buenos Aires, Argentina) and Thermo Fisher Scientific (Buenos Aires, Argentina), and are listed in Table S3. Digestions were achieved using Promega (Biodynamics SRL, Buenos Aires, Argentina) enzymes as necessary. DNA sequencing was performed by Macrogen DNA Sequencing Service (Macrogen Corp., Seoul, South Korea). 
The $B$. diazoefficiens deletion mutants $\Delta$ fli $L_{S}$ and $\Delta f i L_{L}$ were obtained using the unmarked in-frame deletion strategy as described previously (49). To generate $\Delta$ fli $_{s}$ a 249 -bp fragment upstream and a 216-bp fragment downstream of fli $L_{\mathrm{s}}$ were amplified using the primer pairs up5826_Fw/up5826_Rv and dw5826_Fw/dw5826_Rv, respectively. The resultant fragments were gel purified and combined as the template to be amplified with the primer pair up5826_Fw/dw5826_Rv. The 486-bp fragment obtained was cloned into the EcoRI/HindIII sites of pK18mobsacB vector to generate pKsacB::fliLs. The gene replacement was made by mobilization of the construction from S17-1 to B. diazoefficiens USDA 110 by biparental mating, and the simple crossover (cointegrate) was selected by $\mathrm{Km}$ resistance. To induce double crossover recombination, the selected transconjugants were plated in yeast mannitol agar (YMA) supplemented with $10 \%$ (wt/vol) sucrose. The resulting clones were checked by PCR in order to select the mutant genotype, and the correct in-frame deletion was verified by DNA sequencing. Thus, the length of fliLs ORF was reduced in $76.6 \%$. To complement the mutation, the complete bll5826 sequence was amplified from B. diazoefficiens USDA 110 chromosomal DNA, using Pfu DNA polymerase with the primer pair Ext5826_Fw/Ext5826_Rv. The resulting PCR fragment was cloned into the EcoRV site of pBBR1MCS2 to create pBBR::fliL. The construction was checked by PCR and DNA sequencing and then transferred into the desired $B$. diazoefficiens strain by conjugation. The transconjugants were selected by $\mathrm{Km}$ resistance and confirmed by $\mathrm{PCR}$ amplification.

To obtain the $B$. diazoefficiens $\Delta$ flil $L_{L}$ mutant, an upstream fragment of $278 \mathrm{bp}$ and a downstream fragment of 419 bp were amplified using the primer pair up6868_Fw/up6868_Rv and dw6868_Fw/ dw6868_Rv, respectively. These fragments were gel purified and combined as a template to be amplified with the primer pair up6868_Fw/dw6868Rv. The 734-bp fragment obtained was cloned into the EcoRl/Hindlll sites of the pK18mobsacB vector to generate the pKsacB::fliL $L_{L}$ construction. The gene replacement and double-crossover recombination was performed like generation of the $\Delta \mathrm{Alil}_{S}$ deletion described above. Thus, the fli $L_{L}$ deletion comprises $90 \%$ of the wild-type ORF. For $\Delta$ fliL $L_{L}$ complementation, the complete coding sequence of bll6868 was amplified from B. diazoefficiens USDA 110 chromosomal DNA, using Pfu DNA polymerase and the primer pair Ext6868_Fw/Ext6868_Rv. The amplified fragment was cloned into the $\mathrm{Xbal} / \mathrm{Kpnl}$ sites of pFAJ1708 to create pFAJ::fli $L_{L}$. The construction was checked by PCR and DNA sequencing and then mobilized into the desired B. diazoefficiens strain by biparental mating, selected by Tc resistance, and checked by PCR amplification, as before.

The $B$. diazoefficiens $\triangle$ lafA strain was obtained by introducing the pMJA05 plasmid into USDA 110 by conjugation as previously described (4). A double-crossover event causing $\mathrm{Km}$ sensitivity and $\mathrm{Sp}$-Sm resistance was selected. The mutation was checked by flagellins production as previously described (4). The same strategy was used with the $\Delta$ flil $_{s}$ background to obtain the $\triangle$ flil $L_{s} \triangle l a f A$ double mutant strain.

To generate the $B$. diazoefficiens $\triangle$ fliC strain, the pMJA10 plasmid carried by E. coli S17-1 was introduced into USDA 110 by biparental mating as described previously (4). A double-crossover event was selected by searching for $\mathrm{Gm}$ sensitivity and $\mathrm{Km}$ resistance among the transconjugants. The selected clones were checked by flagellin production as described previously (4). The same approach was used with the background of $\triangle A l i L_{L}$ and $\triangle l a f A$ to obtain the $\triangle f l i L, \triangle f l i C$ and $\triangle$ lafA $\triangle$ fliC double mutant strains, respectively.

The E. coli MG1655 $\Delta$ fliL mutant was generated by P1 transduction of the fliL::kan mutation from strain JW1981 (E. coli Genetic Stock Center) into wild-type MG1655, before curing the resistance cassette using the recombinase activity of $\mathrm{pCP} 20$ (50). The strain was verified by PCR using appropriate flanking primers.

For $B$. diazoefficiens fliL heterologous complementation, the entire ORFs of fliL $L_{S}$ and fliL $L_{L}$ were amplified from $B$. diazoefficiens USDA 110 chromosomal DNA using the primer pairs fli $\mathrm{L}_{s_{-}} \mathrm{Fw} / \mathrm{fli} \mathrm{L}_{\mathrm{s}_{-}} \mathrm{Rv}$ and fli $L_{L} F w / f l i L_{L} R$, respectively. Then, both fragments were cloned into pBluescriptSK(+) to generate plasmids pBlue::ffil $L_{S}$ and pBlue::ffil $L_{L}$, then checked by PCR and sequencing. For cloning into pBAD24 expression plasmids, the entire ORFs were amplified from both pBlue::fliL background using PCR and appropriate primers engineered to include Nhel and Hindlll restriction sites in forward and reverse primers, respectively (5826_EC_Fw/5826_Ec_Rv for fliL $L_{S}$ and 6868_EC_Fw/6868_Ec_Rv for fliL $L_{L}$ ). Final constructs, named pBAD::fli $L_{S}$ and $\mathrm{pBAD}:: f_{i L} L_{L}$, were confirmed by DNA sequencing after cloning and moved to $E$. coli MG1655 $\Delta$ fliL by electroporation and further selection of ampicillin resistance clones (23).

Motility measurements. For B. diazoefficiens swimming assay in semisolid medium, fresh bacteria were inoculated using a sterile toothpick on Götz minimal medium supplemented with $0.5 \%(\mathrm{wt} / \mathrm{vol})$ mannitol and $0.3 \%(\mathrm{wt} / \mathrm{vol})$ agar or PSY-arabinose $0.3 \%(\mathrm{wt} / \mathrm{vol})$ agar, and the motility halo was registered as described (9). To increase medium viscosity, polyvinylpyrrolidone (PVP) K-90 was added to the motility plates as described (2). E. coli MG1655 swimming and swarming experiments were performed as described (23) and included the wild type and fliL mutant, both carrying PBAD24 empty vector, with the E. coli $\Delta$ fliL complemented strain included as a positive control (23). Plates were supplemented with $0.5 \%$ arabinose (wt/vol) instead of glucose, to induce vector expression. B. diazoefficiens cells used for swimming speed measurements were grown in HMY (5) supplemented with arabinose $0.5 \%$ (wt/vol) until an optical density at $500 \mathrm{~nm}\left(\mathrm{OD}_{500}\right)$ of 0.5 at $28^{\circ} \mathrm{C}$ and $60 \mathrm{rpm}$ was reached and then were diluted 1:2 in $\mathrm{HM}$ salts and incubated at $28^{\circ} \mathrm{C}$ without shaking for $4 \mathrm{~h}$ before microscopic observation. This $4-\mathrm{h}$ incubation increased the motility fraction, and the incubated culture was again diluted 1:25 to be observed under the microscope. To evaluate the PVP effect, samples from 1:2 dilution and $4 \mathrm{~h}$ of incubation were gently diluted in HM salts (1:25) containing different concentrations of PVP added just before observation. Free-swimming cells were observed under high-intensity dark-field microscopy (Olympus BX50) and recorded at 1/30-s (30 frames per second) (Sony Exwave HAD camera). Straight-line speeds were then analyzed using Move-tr/2D software (Library, Tokyo, Japan).

Flagellin preparation and analysis. Cells were grown in $\mathrm{HMY}$ medium with $0.5 \%(\mathrm{wt} / \mathrm{vol})$ arabinose or $0.5 \%$ (wt/vol) mannitol as the sole carbon source (5) until saturation, or in PSY medium with $0.1 \%$ 
arabinose (41) until an $\mathrm{OD}_{500}$ of 0.6 . Then, the cultures were kept on ice for $15 \mathrm{~min}$, vortexed vigorously for $4 \mathrm{~min}$, and centrifuged at $13,520 \times \mathrm{g}$ for $30 \mathrm{~min}$ at $4^{\circ} \mathrm{C}$. The supernatants were incubated with $1.3 \%$ (vol/vol) polyethylene glycol 6000 and $2.5 \mathrm{M} \mathrm{NaCl}$ from $2 \mathrm{~h}$ to overnight at $4^{\circ} \mathrm{C}$. After that, the suspension was centrifuged at $13,520 \times g$ for $45 \mathrm{~min}$ at $4^{\circ} \mathrm{C}$, and the pellets were resuspended in Laemmli loading buffer (51) or double-distilled water, as needed. To perform the analysis, samples were boiled for $10 \mathrm{~min}$, centrifuged at $14,000 \times \mathrm{g}$ for $10 \mathrm{~min}$, and separated by $12.5 \%$ sodium dodecyl sulfate polyacrylamide gel electrophoresis (51). Protein quantification was performed using the Bradford method (52).

RNA extraction and retrotranscribed PCR. Total RNA extraction was performed from cells cultured in liquid and semisolid PSY-arabinose media. From liquid cultures, B. diazoefficiens cells were grown until an $\mathrm{OD}_{500}$ of 0.6 , placed for $5 \mathrm{~min}$ on ice, and centrifuged for $25 \mathrm{~min}$ at $11,180 \times g$ at $4^{\circ} \mathrm{C}$. Immediately after, we proceeded with the disruption of the cells to extract their RNA as previously described (5).

From semisolid medium, bacteria were inoculated onto soft agar plate and incubated at $28^{\circ} \mathrm{C}$ for 13 days. At that time, when the diameter of the motility halo was approximately $5.5 \mathrm{~cm}$, cells were isolated from the whole motility halo $(\mathrm{WMH})$ or from the border of the motility halo (BMH). The BMH was defined as the more external motility ring in a radius of $1.5 \mathrm{~cm}$ (see the experimental scheme in the supplemental material). In both cases, samples were taken with a clean spoon and placed into a $50-\mathrm{ml}$ plastic tube with an equal volume of double-distilled water. After 5 min on ice, the samples were vortexed for $1 \mathrm{~min}$ and centrifuged for $25 \mathrm{~min}$ at $11,180 \times g$ at $4^{\circ} \mathrm{C}$. Then, the agar above the cells was carefully separated from them to continue with the cell disruption as described above (see the experimental scheme in the supplemental material). To check the quality of CDNA preparations, PCRs were performed with the primer pair q5843_Fw/q5843_Rv. The absence of contaminating DNA was demonstrated by the lack of PCR amplification in an RNA sample that was not subjected to reverse transcription. As a constitutive control, primers for the housekeeping gene sigA were used (53).

Quantitative PCR. The CDNAs were amplified using the primer pairs lafR_Fw/q6846int_Rv, q6861_Fw/q6861_Rv, q6864-2_Fw/q6864-2_Rv, and q6868_Fw/q6868_Rv. The reactions were performed using iQ SYBR green Supermix (Bio-Rad, USA) according to the manufacturers' instructions. Normalized expression values were calculated as the ratio between the relative quantities of the gene of interest (GOI) and the relative quantities of the $\operatorname{sig} A$ housekeeping gene (53).

Transmission electron microscopy. To obtain samples from swimming plates, a piece of soft agar with bacteria was taken using the reverse end of a yellow tip, submerged in $50 \mu \mathrm{l}$ of double-distilled water, and mixed gently. A drop of this suspension was placed on 200-mesh copper grid that had been coated with collodion to proceed as previously described (2).

\section{SUPPLEMENTAL MATERIAL}

Supplemental material is available online only.

SUPPLEMENTAL FILE 1, PDF file, 0.8 MB.

\section{ACKNOWLEDGMENTS}

We thank S. Jurado, R. Peralta and K. Maki for help with electron microscopy and Rasika Harshey for support.

This work was supported by the Agencia Nacional de Promoción de la Investigación Científica y Tecnológica (ANPCyT), by the Bec.Ar program of Ministerio de Educación, Cultura, Ciencia y Tecnología, and by Consejo Nacional de Investigaciones Científicas y Técnicas (CONICET), Argentina. E.J.M., J.I.Q., M.J.A., and A.R.L. are members of the Scientific Career of CONICET. F.M. is a fellow of CONICET.

The funders had no role in study design or data collection and interpretation. We declare that we have no conflict of interests.

\section{REFERENCES}

1. Liu R, Ochman H. 2007. Origins of flagellar gene operons and secondary flagellar systems. J Bacteriol 189:7098-7104. https://doi.org/10.1128/JB .00643-07.

2. Quelas Jl, Althabegoiti MJ, Jimenez-Sanchez C, Melgarejo AA, Marconi VI, Mongiardini EJ, Trejo SA, Mengucci F, Ortega-Calvo JJ, Lodeiro AR. 2016. Swimming performance of Bradyrhizobium diazoefficiens is an emergent property of its two flagellar systems. Sci Rep 6:23841. https://doi.org/10 .1038/srep23841.

3. Garrido-Sanz D, Redondo-Nieto $M$, Mongiardini $E$, Blanco-Romero $E_{\text {, }}$ Durán D, Quelas Jl, Martin M, Rivilla R, Lodeiro AR, Althabegoiti MJ. 2019. Phylogenomic analyses of Bradyrhizobium reveal uneven distribution of the lateral and subpolar flagellar systems, which extends to Rhizobiales. Microorganisms 7:50. https://doi.org/10.3390/microorganisms7020050.

4. Althabegoiti MJ, Covelli JM, Pérez-Giménez J, Quelas Jl, Mongiardini EJ, López MF, López-García SL, Lodeiro AR. 2011. Analysis of the role of the two flagella of Bradyrhizobium japonicum in competition for nodulation of soybean. FEMS Microbiol Lett 319:133-139. https://doi.org/10.1111/j .1574-6968.2011.02280.x.

5. Mongiardini EJ, Quelas JI, Dardis C, Althabegoiti MJ, Lodeiro AR. 2017. Transcriptional control of the lateral-flagellar genes of Bradyrhizobium diazoefficiens. J Bacteriol 199:e00253-17.

6. Merino S, Tomás JM. 2009. Lateral flagella systems, p 173-190. In Jarrell KF (ed), Pili and flagella: current research and future trends. Caister Academic Press, Poole, United Kingdom.

7. Partridge JD, Harshey RM. 2013. Swarming: flexible roaming plans. J Bacteriol 195:909-918. https://doi.org/10.1128/JB.02063-12.

8. Kanbe M, Yagasaki J, Zehner S, Gottfert M, Aizawa S. 2007. Characterization of two sets of subpolar flagella in Bradyrhizobium japonicum. J Bacteriol 189:1083-1089. https://doi.org/10.1128/JB.01405-06.

9. Althabegoiti MJ, López-García SL, Piccinetti C, Mongiardini EJ, PérezGiménez J, Quelas Jl, Perticari A, Lodeiro AR. 2008. Strain selection for improvement of Bradyrhizobium japonicum competitiveness for nodula- 
tion of soybean. FEMS Microbiol Lett 282:115-123. https://doi.org/10 .1111/j.1574-6968.2008.01114.x.

10. Bubendorfer S, Koltai M, Rossmann F, Sourjik V, Thormann KM. 2014 Secondary bacterial flagellar system improves bacterial spreading by increasing the directional persistence of swimming. Proc Natl Acad Sci U S A 111:11485-11490. https://doi.org/10.1073/pnas.1405820111.

11. Covelli JM, Althabegoiti MJ, Lopez MF, Lodeiro AR. 2013. Swarming motility in Bradyrhizobium japonicum. Res Microbiol 164:136-144. https://doi.org/10.1016/j.resmic.2012.10.014.

12. Cogo C, Perez-Gimenez J, Rajeswari CB, Luna MF, Lodeiro AR. 2018. Induction by Bradyrhizobium diazoefficiens of different pathways for growth in D-mannitol or L-arabinose leading to pronounced differences in $\mathrm{CO}_{2}$ fixation, $\mathrm{O}_{2}$ consumption, and lateral-flagellum production. Front Microbiol 9:1189. https://doi.org/10.3389/fmicb.2018.01189.

13. Fernández N, Cabrera JJ, Varadarajan AR, Lutz S, Ledermann R, Roschitzki B, Eberl L, Bedmar EJ, Fischer H-M, Pessi G, Ahrens CH, Mesa S. 2019. An integrated systems approach unveils new aspects of microoxiamediated regulation in Bradyrhizobium diazoefficiens. Front Microbiol 10:924-924. https://doi.org/10.3389/fmicb.2019.00924.

14. Pallen MJ, Matzke NJ. 2006. From the origin of species to the origin of bacterial flagella. Nat Rev Microbiol 4:784-790. https://doi.org/10.1038/ nrmicro1493.

15. Terashima H, Kawamoto A, Morimoto YV, Imada K, Minamino T. 2017. Structural differences in the bacterial flagellar motor among bacterial species. Biophys Physicobiol 14:191-198. https://doi.org/10.2142/biophysico.14 .0_191.

16. Purcell EM. 1977. Life at low Reynolds number. Am J Phys 45:3-11. https://doi.org/10.1119/1.10903.

17. Motaleb MA, Pitzer JE, Sultan SZ, Liu J. 2011. A novel gene inactivation system reveals altered periplasmic flagellar orientation in a Borrelia burgdorferi fliL mutant. J Bacteriol 193:3324-3331. https://doi.org/10 .1128/JB.00202-11.

18. Moon KH, Zhao X, Manne A, Wang J, Yu Z, Liu J, Motaleb MA. 2016. Spirochetes flagellar collar protein FlbB has astounding effects in orientation of periplasmic flagella, bacterial shape, motility, and assembly of motors in Borrelia burgdorferi. Mol Microbiol 102:336-348. https://doi .org/10.1111/mmi.13463.

19. Kumar A, Isumi M, Sakuma M, Zhu S, Nishino Y, Onoue Y, Kojima S, Miyanoiri Y, Imada K, Homma M. 2017. Biochemical characterization of the flagellar stator-associated inner membrane protein FliL from Vibrio alginolyticus. J Biochem 161:331-337. https://doi.org/10.1093/jb/ mvw076.

20. Takekawa N, Isumi M, Terashima H, Zhu S, Nishino $Y$, Sakuma M, Kojima S, Homma M, Imada K, Takekawa N, Isumi M, Terashima H, Zhu S, Nishino Y, Sakuma M, Kojima S, Homma M, Imada K. 2019. Structure of Vibrio FliL, a new stomatin-like protein that assists the bacterial flagellar motor function. mBio 10:e00292-19. https://doi.org/10.1128/mBio.00292-19.

21. Belas R. 2014. Biofilms, flagella, and mechanosensing of surfaces by bacteria. Trends Microbiol 22:517-527. https://doi.org/10.1016/j.tim .2014.05.002.

22. Attmannspacher U, Scharf BE, Harshey RM. 2008. FliL is essential for swarming: motor rotation in absence of FliL fractures the flagellar rod in swarmer cells of Salmonella enterica. Mol Microbiol 68:328-341. https:// doi.org/10.1111/j.1365-2958.2008.06170.x.

23. Partridge JD, Nieto $V$, Harshey RM. 2015. A new player at the flagellar motor: FliL controls both motor output and bias. mBio 6:e02367. https:// doi.org/10.1128/mBio.02367-14.

24. Zhu S, Kumar A, Kojima S, Homma M. 2015. FliL associates with the stator to support torque generation of the sodium-driven polar flagellar motor of Vibrio. Mol Microbiol 98:101-110. https://doi.org/10.1111/mmi.13103.

25. Lin TS, Zhu S, Kojima S, Homma M, Lo CJ. 2018. FliL association with flagellar stator in the sodium-driven Vibrio motor characterized by the fluorescent microscopy. Sci Rep 8:11172. https://doi.org/10.1038/s41598 -018-29447-x.

26. Tipping MJ, Delalez NJ, Lim R, Berry RM, Armitage JP, Tipping MJ, Delalez NJ, Lim R, Berry RM, Armitage JP. 2013. Load-dependent assembly of the bacterial flagellar motor. mBio 4:e00551-13. https://doi.org/10.1128/ mBio.00551-13.

27. Belas R, Suvanasuthi R. 2005. The ability of Proteus mirabilis to sense surfaces and regulate virulence gene expression involves FliL, a flagellar basal body protein. J Bacteriol 187:6789-6803. https://doi.org/10.1128/ JB.187.19.6789-6803.2005

28. Cusick K, Lee YY, Youchak B, Belas R. 2012. Perturbation of FliL interferes with Proteus mirabilis swarmer cell gene expression and differentiation. J Bacteriol 194:437-447. https://doi.org/10.1128/JB.05998-11.

29. Lee YY, Patellis J, Belas R. 2013. Activity of Proteus mirabilis FliL is viscosity dependent and requires extragenic DNA. J Bacteriol 195: 823-832. https://doi.org/10.1128/JB.02024-12.

30. Chawla R, Ford KM, Lele PP. 2017. Torque, but not FliL, regulates mechanosensitive flagellar motor-function. Sci Rep 7:5565. https://doi .org/10.1038/s41598-017-05521-8.

31. Lee YY, Belas R. 2015. Loss of FliL alters Proteus mirabilis surface sensing and temperature-dependent swarming. J Bacteriol 197:159-173. https:// doi.org/10.1128/JB.02235-14.

32. Fabela S, Domenzain C, De la Mora J, Osorio A, Ramirez-Cabrera V, Poggio S, Dreyfus G, Camarena L. 2013. A distant homologue of the FlgT protein interacts with MotB and FliL and is essential for flagellar rotation in Rhodobacter sphaeroides. J Bacteriol 195:5285-5296. https://doi.org/ 10.1128/JB.00760-13.

33. Jenal U, White J, Shapiro L. 1994. Caulobacter flagellar function, but not assembly, requires FliL, a non-polarly localized membrane protein present in all cell types. J Mol Biol 243:227-244. https://doi.org/10.1006/jmbi .1994.1650.

34. Kaneko T, Nakamura Y, Sato S, Minamisawa K, Uchiumi T, Sasamoto S, Watanabe A, Idesawa K, Iriguchi M, Kawashima K, Kohara M, Matsumoto M, Shimpo S, Tsuruoka H, Wada T, Yamada M, Tabata S. 2002. Complete genomic sequence of nitrogen-fixing symbiotic bacterium Bradyrhizobium japonicum USDA110. DNA Res 9:189-197. https://doi.org/10.1093/ dnares/9.6.189.

35. Wolfe AJ, Berg HC. 1989. Migration of bacteria in semisolid agar. Proc Natl Acad Sci U S A 86:6973-6977. https://doi.org/10.1073/pnas.86.18.6973.

36. Götz R, Limmer N, Ober K, Schmitt R. 1982. Motility and chemotaxis in two strains of Rhizobium with complex flagella. J Gen Microbiol 128: 789-798. https://doi.org/10.1099/00221287-128-4-789.

37. McCarter L, Hilmen M, Silverman M. 1988. Flagellar dynamometer controls swarmer cell differentiation of V. parahaemolyticus. Cell 54:345-351. https://doi.org/10.1016/0092-8674(88)90197-3.

38. Adler J. 1966. Chemotaxis in bacteria. Science 153:708-716. https://doi .org/10.1126/science.153.3737.708.

39. Iturralde ET, Covelli JM, Alvarez F, Perez-Gimenez J, Arrese-Igor C, Lodeiro AR. 2019. Soybean-nodulating strains with low intrinsic competitiveness for nodulation, good symbiotic performance, and stresstolerance isolated from soybean-cropped soils in Argentina. Front Microbiol 10:1061. https://doi.org/10.3389/fmicb.2019.01061.

40. Vincent JM. 1970. A manual for the practical study of the root nodule bacteria. IBP handbook no. 15. Blackwell Scientific Publications, Oxford, United Kingdom.

41. Regensburger B, Hennecke H. 1983. RNA polymerase from Rhizobium japonicum. Arch Microbiol 135:103-109. https://doi.org/10.1007/bf00408017.

42. Sambrook J, Russell D. 2001. Molecular cloning: a laboratory manual, 3rd ed. Cold Spring Harbor Laboratory Press, New York, NY.

43. Cole MA, Elkan GH. 1973. Transmissible resistance to penicillin G, neomycin, and chloramphenicol in Rhizobium japonicum. Antimicrob Agents Chemother 4:248-253. https://doi.org/10.1128/aac.4.3.248.

44. Dehal PS, Joachimiak MP, Price MN, Bates JT, Baumohl JK, Chivian D, Friedland GD, Huang KH, Keller K, Novichkov PS, Dubchak IL, Alm EJ, Arkin AP. 2010. MicrobesOnline: an integrated portal for comparative and functional genomics. Nucleic Acids Res 38:D396-400. https://doi .org/10.1093/nar/gkp919.

45. Tsirigos KD, Peters C, Shu N, Kall L, Elofsson A. 2015. The TOPCONS web server for consensus prediction of membrane protein topology and signal peptides. Nucleic Acids Res 43:W401-407. https://doi.org/10 $.1093 /$ nar/gkv485

46. Sonnhammer EL, von Heijne G, Krogh A. 1998. A hidden Markov model for predicting transmembrane helices in protein sequences. Proc Int Conf Intell Syst Mol Biol 6:175-182.

47. Kumar S, Stecher G, Li M, Knyaz C, Tamura K. 2018. MEGA X: Molecular Evolutionary Genetics Analysis across computing platforms. Mol Biol Evol 35:1547-1549. https://doi.org/10.1093/molbev/msy096.

48. Le SQ, Gascuel O. 2008. An improved general amino acid replacement matrix. Mol Biol Evol 25:1307-1320. https://doi.org/10.1093/molbev/ msn067.

49. Sukdeo N, Charles TC. 2003. Application of crossover-PCR-mediated deletion-insertion mutagenesis to analysis of the $b d h A-x d h A 2-x d h B 2$ mixed-function operon of Sinorhizobium meliloti. Arch Microbiol 179: 301-304. https://doi.org/10.1007/s00203-003-0532-9.

50. Datsenko KA, Wanner BL. 2000. One-step inactivation of chromosomal 
genes in Escherichia coli K-12 using PCR products. Proc Natl Acad Sci U S A 97:6640-6645. https://doi.org/10.1073/pnas.120163297.

51. Laemmli UK. 1970. Cleavage of structural proteins during the assembly of the head of bacteriophage T4. Nature 227:680-685. https://doi.org/ 10.1038/227680a0.

52. Bradford MM. 1976. A rapid and sensitive method for the quantitation of microgram quantities of protein utilizing the principle of protein-dye binding. Anal Biochem 72:248-254. https://doi.org/10.1006/abio.1976 .9999.

53. Hauser F, Lindemann A, Vuilleumier S, Patrignani A, Schlapbach $R$ Fischer HM, Hennecke H. 2006. Design and validation of a partialgenome microarray for transcriptional profiling of the Bradyrhizobium japonicum symbiotic gene region. Mol Genet Genomics 275:55-67. https://doi.org/10.1007/s00438-005-0059-7. 